\title{
Context Inference Using Correlation in Human Behaviour
}

\author{
Dušan Zeleník \\ Faculty of Informatics and Information Technologies \\ Slovak University of Technology \\ Bratislava, Slovakia \\ zelenik@fiit.stuba.sk
}

\author{
Mária Bieliková \\ Faculty of Informatics and Information Technologies \\ Slovak University of Technology \\ Bratislava, Slovakia \\ bielik@fiit.stuba.sk
}

\begin{abstract}
Context-aware information retrieval received a significant amount of attention last years. This paper addresses some of the challenges in context acquisition. It is focused on a method for inference of unavailable contextual information using machine learning. The method for context inference is based on observed behaviour of individual user and virtual communities of similar users. We work with with contextual information such as location, time or weather in the domain of news recommending. We discuss the role of user behaviour and its significant impact on the actual information need, which directly influences information retrieval or recommendation process. In experiments we demonstrate the impact of inferred context on the recommendation process and its precision and recall. We use behaviour of news readers to predict their interest in news. We present context-aware recommendation which is supported by our method for context inference.
\end{abstract}

Keywords-context, behaviour, machine learning, prediction

\section{INTRODUCTION}

The availability of contextual information through mobile devices leads to spread of advanced methods for satisfying user needs such as personalized recommendation or adaptive navigation. The context is understood as a set of conditions describing the user and her state in the environment [1]. However, complex environment surrounding the user is poorly described and contextual information is difficult to acquire in some cases. For instance, we are able to obtain location for a user whose mobile device is capable of receiving GPS signal. On the other hand, the same user might disallow the device to retrieve geo-location due to various reasons (privacy issues, battery consumption). Thus we want to solve this problem and infer the context implicitly by mining it from available data.

Our aim is based on the cognitive psychology and research [2] which observed the effort of human being to recognize emotions of another human. Humans use various contextual information to recognize the facial expression and classify it. We reproduced this observation and managed to infer the context using other contextual information. In our method for context inference we analyse behaviour of several users and we recognize virtual communities by behavioural similarities of users. Knowing communities of similar users allows to infer the context where it is missing.
We decided to use combination of available data mining techniques to achieve this. Our method for context inference works with continuous values. We present this in contrast with discretization which is used in association rules mining.

In this paper we show that by context inference we obtain new valuable contextual information respecting case-based reasoning. Another motivation is to use inferred context to improve the quality of existing algorithms for the recommendation systems. For this purpose we use the data from SME.sk which contains logs of news reading and articles which were published.

We structured the paper to present our observations, our proposal and experiments. In section III we present our findings which inspired us to design our method for context inference. This method is presented in detail in the section IV. This section is followed by our experiments (section V) which which we made with aforementioned dataset. Our future work and contributions are discussed in the last section.

\section{RELATED WORK}

Many recommender systems use context as a source for mining the interest of users [3]. However context-aware recommender systems are often based on different technique due to poor performance [4] of purely context based recommender systems. We recognized that huge sparsity in contextual information could be one reason why are not context-based systems successful. We identified that by reducing the sparsity we should be able to increase the precision.

Kahng et al. [5] work with the context which is predefined. They are modelling the domain and context. Context is then used as a factor for ranking the items. As an example they present weather and its impact on the user needs. They predefined the relation between context and temporal interest in song listening. In specific domains is some contextual information more relevant than the rest. It requires to analyse the domain and obtain a knowledge which could be used. Baltranus et al. [6] research the relevance of the context in the system by asking the user directly. Supposed context has positive impact on the success of their method, however, Asoh et al. [7] proves that there is a significant difference 
between the real and supposed context and its perception of the user. In both cases it is an explicit form of the context acquisition. The main problem here is that we can not engage everyone into giving of the explicit feedback. This only proves that acquisition of some contextual information is a problem in many domains and need to be solved or we have to find alternatives to acquire context.

One option is to use machine learning to infer context where its missing. We actually need a set of contextual information which has to be available in order to infer context. Riboni et al. [8] announced a hybrid of statistical approach and ontological reasoning in order to infer the context. Further utilization in the COSAR project shows improvement by combining both of these approaches. Our approach is also based on statistics and boosted by empirically observed effects of correlation in users' behaviour. The correlation of the behaviour is the correlation of the contextual information in other words. For instance, Konomi et al. [9] presented connections between people formed by co-presence at places. These connections are based on geo-location but what is more interesting, geolocation correlates with social connections among users.

Cadiz et al. [10] or Rahnama et al. [11] and their work enables us to work with more users and in various systems. Unifying context-aware systems into frameworks enables us to gather usage logs more globally. For example, contextual information linked with user actions from various systems improves our chances to infer missing context. However, including information on the past, present and future context [12] we increase the complexity of such an approach. This is because of quantity of information which are represented and its multiplication in aforementioned manner. In fact, many researchers address their effort to reduce the complexity of context aware recommender system [13].

\section{CONTEXT Influence ON Human Behaviour}

The context is typically understood as a set of conditions describing a user and her state in the environment [1]. The context is always present when user makes decisions. For instance, context could be weather, time (and its derivations like day of week or part of day), mood, location etc. We observed the impact of the context on user behaviour by simple news reading analysis. User behaviour, which we reference as a set of actions is influenced by a set of conditions. By action in the news reading domain, we understand clicks. Each click has its attributes such as timestamp, target article and user identifier. Using these actions and associated contextual information we discover rules which could be applied to predict user behaviour.

We work with the dataset of user actions and articles from the news portal SME.sk. We are working with real users and articles which are published every day. Every day around 250 new articles are added. These articles are read

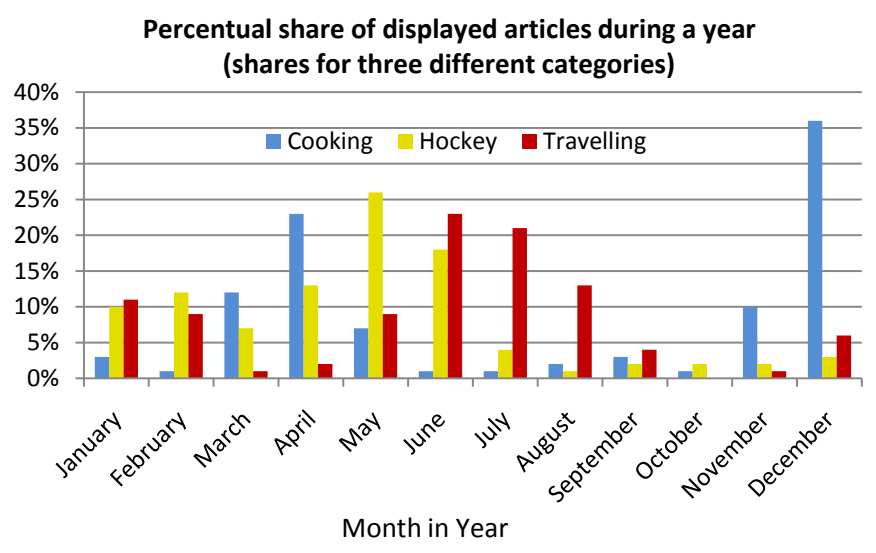

Figure 1. Readers are influenced by forthcoming events. We can observe the impact as an increase of displayed articles on specific topics (monitoring real users at SME.sk).

by roughly 40 thousands readers, what generates around 80 thousands clicks per day (displayed articles only).

From the user and her actions, we continue to research the group of users and their actions [14]. Not only individual user has behavioural patterns. We know that also behaviour of the masses emerges into pattern. Analyses of the news reading during a year showed that readers are influenced by the context of time, particularly by forthcoming events (see Figure 1).

Improvement of the behaviour prediction of SME.sk readers and users in general is one of our key contributions of this paper. Behaviour of the mass or isolated group could be influenced by individuals in the group. There are always authorities or hubs in the group who are leading the behaviour of the rest of the group [15]. Our generic approach could work with any type of action taken by the user. What we do is add contextual information to every action which already contains user identifier and subject identifier.

Some actions of users correlate in the consideration of the context. This effect is very important for the context inference. Our intent is to find similar users according to their behaviour. We propose to discover the user similarity by searching the similarities using vectors of represented user actions (see Figure 2).

We propose the way of calculating similarity to support different weights of context components. Weighting the components could be useful in experimentation where we could identify different relevance of context factors [6] and reduce ineligible noise of unnecessary context factors. Real world employment of our approach does not require weights because ineligible noise is eliminated automatically by searching for similar users and thus reducing the complexity of the data. 


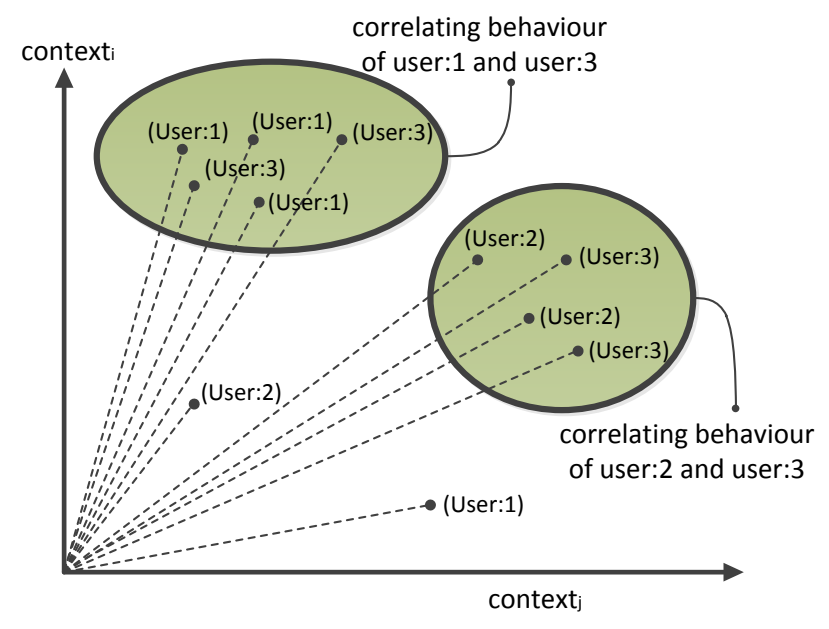

Figure 2. More user actions with contextual information are illustrated in two-dimensional space. We can observe the Euclidean distance of some actions. Theses actions were taken by different users but there are hallmark similarities in particular cases. In this particular example, user:1 and user:2 are similar to user:3 but not to each other.

\section{Method for Context Inference}

We proposed a method for context inference which semantically enriches logs of news reading. The aim of our research is not to acquire easily available context but to infer context components which are not available directly. We work with visible and hidden contextual information. We consider the availability for specific user. Some contextual information is easily acquired some is not. However, it could differ from domain to domain. It means that we can not define exact context type to be visible or hidden. We can split context in specific domain into two types.

- Visible. Available user information which is easily acquired. For instance, time is contextual information which we can easily acquire almost in every domain.

- Hidden. Present but not available. This type of contextual information is not easily acquired. It actually exists and has impact on the user behaviour but we can not obtain this information directly. For example, it could be dwelling time. It definitely affects our needs when we are at home, but system has no direct information on such context.

We want to focus on hidden contextual information. We want to infer context which could not be directly acquired. If context is hidden for specific user it does not mean that it is hidden for other users. From this point of view we can split contextual information hidden for specific user into two types.

- Known. Visible for other users but hidden for the specific user until inferred. For example, when the user reads mostly local news and we can not acquire her location we could infer this context. We can do this through other users with visible context of location who are interested in the same news.

- Unknown. Not visible for anyone. This could be inferred as the constellation of visible context components. Actually we can not recognize this type of context as rigid information. We can only work with the set of other contextual information which describes this type of hidden context.

Context is visible for specific user when it is directly available (using GPS module for location or explicit question to discover mood). Hidden context is affecting the user but we can not acquire it directly. Known context exists for other users but we can not directly acquire this context for specific user (eg. GPS module is disabled or she does not answer questions on her mood). In this paper we focus on hidden context which marked as known.

\section{A. Acquiring Visible Context}

Each action which has been recorded is enriched by visible context components. We use external services such as Wunderground ${ }^{1}$ for weather information, TagTheNet ${ }^{2}$ for keywords and content analyses or IP2Location ${ }^{3}$ for the location. We also process some contextual information with aim to transform specific data to more abstract representation. We process timestamp to have more time context components. We work with values representing minute in the hour, hour in the day, day in the week, week in the month and month in the year. We presumed that there are no complex intervals for human habits. Representations which we named here are empirically chosen to cover all human habits [16].

IP addresses are translated into the country, city and GPS coordinates. Considering locations, we propose algorithm which takes time and IP address for all user actions. We determine whether the action was taken at home or at work. This is done by simple assessment of a place by analysing of the repeated IP address usage during work hours (work days from $8 \mathrm{AM}$ to $17 \mathrm{AM}$ ).

Weather is represented as the value describing the situation from the very bad to very good weather condition and nominal attributes representing conditions ( cloudy, raining, snowing.

Finally, the content is processed as a text while we use the dataset of news reading as an example of the customization. The contextual information is then represented as keywords, section and category in the news classification system.

Our method is not constrained to work only with this contextual information. We designed our method as a generic solution for any domain. We claim that using information

\footnotetext{
${ }^{1}$ http://www.wunderground.com

${ }^{2}$ http://tagthe.net

${ }^{3}$ http://www.ip2location.com
} 
which could be easily acquired (visible context) for one user helps to infer context not easily available (hidden) for another.

\section{B. Discovering Similar Users}

We propose an algorithm for discovering similar users based on contextual data. It is based on collaborative filtering approaches. Collaborative filtering is widely used in recommender systems [17]. The idea is in propagating items between similar users. In our case, we do not use item as a factor of similarity. Context is the only feature used here to discover similar users (see Figure 2). We work with user actions which are represented as vectors. Each vector contains user identifier, identifier of the item associated with the user's action and contextual information which is available. Following steps are applied:

1) Cluster vectors, ignoring user and item identifier. For clustering, we only use the contextual information. We use k-means where vectors are normalized to include only values from interval $\langle 0,1\rangle$. We solve the variance of contextual information by skipping missing components of the vector. Since vectors are normalized we use Euclidean distance as originally defined for k-means.

$$
\operatorname{sim}(p, q)=\sqrt{\left(p_{1}-q_{1}\right)^{2}+\cdots+\left(p_{n}-q_{n}\right)^{2}}
$$

2) Calculate the rate of users co-occurrence in clusters. Every action belongs to user therefore every user has her action in more clusters. To calculate similarity among users we propose the equation

$$
\operatorname{sim}(u, v)=\frac{\sum_{i=0}^{n}\left(\left|U \cap C_{i}\right|+\left|V \cap C_{i}\right|\right)}{|U|+|V|}
$$

where $\mathrm{U}$ and $\mathrm{V}$ are sets of actions for users $\mathrm{u}$ and $\mathrm{v}$ and $\mathrm{C}$ is the set of clusters.

3) Sort users by their similarity. Calculation of similarity gives us a value from the interval $\langle 0,1\rangle$ for each pair of users. Greater values indicates greater similarity.

\section{Hidden Context Inference}

Hidden context, from our definition is visible for other users. We exploit the effect of correlations in users' behaviour and infer hidden context using following algorithm. We represent user's actions as an input vector. Input vector contains only available contextual information which had been acquired. The vector could be also fabricated and used as a query to infer context for supposed situation. Hidden context inference is done in following steps:

1) Filter history using user similarity. Since we know similar users (see Section IV-B), we are able to filter unnecessary actions and reduce the complexity of further computation. Records of users actions are filtered and only relevant actions are kept.

2) Find similar actions. Searching for most similar vectors is based on computation of Euclidean similarity (see Equation (1)).

3) Enrich action by hidden context. The set of most similar vectors contain contextual information which was not available for original input vector.

$$
\text { hidden }=I \cup\left(\bigcup_{i=0}^{n} A_{i}\right)
$$

where $I$ is a set of contexts for input action and $A_{i}$ is the set of contexts for similar users' actions.

\section{EXPERIMENTS}

We show two experiments with our method and dataset of news reading from SME. sk. We work with activity logs of 500 users who were active in news reading for one month. They used mobile devices, notebook or personal computers. However, we can identify unique device only, not user, since cookie is used as the only identifier at the moment. Another drawback is that users do not express explicit feedback on the displayed article. We have only information about the action of displaying the article. Using this dataset we focus on two hypotheses and prove our concept.

We presume that the inferred information correctly enriches existing data. We proved this presumption by inferring context which is visible. We compared inferred and real contextual information and calculated relative error, precision and recall.

We presume that inferred contextual information improves the prediction of user activity. We repeat the same prediction with and without inferred context and compare the results.

\section{A. Context Inference for Readers}

We conducted this experiment as inference of visible context. Firstly, we deleted this information and in the next step we used our method to infer it. Then we compared original information with the inferred one. Observed results are shown in the Table I. We present relative error calculated as deviance from the original data. All numeric and nominal attributes are normalized using interval $<0,1\rangle$. We also made the calculation of precision and recall where we took predicted attributes as retrieved items and original attributes as relevant items. Matching items have to fulfil the condition of maximal allowed error which we defined to be 0.1 .

Experiments are grouped by the superclass of the context type. We discuss the context of place (whether user is at home, at work or out). Context of time specifies when the user performed some action (relative to minute of hour, hour of day, day of week etc.). Geo-location consists of city, country and GPS coordinates. Context of content (category and section of news classification system for SME.sk 
Table I

SUCCESS OF CONTEXT INFERENCE. WE PRESENT CONTEXT OF PLACE (HOME, WORK, OTHER), CONTEXT OF TIME (HOUR OF DAY, DAY OF WEEK, WEEK OF MONTH), CONTEXT OF GEO-LOCATION (CITY, STATE) AND CONTENT (CATEGORY, SECTION)

\begin{tabular}{|c|c|c|c|c|}
\hline context & relative error & recall & precision & f-measure \\
\hline place & 0.118 & $31 \%$ & $57 \%$ & $40 \%$ \\
time & 0.096 & $40 \%$ & $73 \%$ & $51 \%$ \\
geo-location & 0.082 & $50 \%$ & $96 \%$ & $66 \%$ \\
content & 0.031 & $58 \%$ & $94 \%$ & $72 \%$ \\
\hline
\end{tabular}

Table II

PREDICTING BEHAVIOUR OF THE READER.

\begin{tabular}{|c|c|c|c|}
\hline contextual information & recall & precision & f-measure \\
\hline original & $41 \%$ & $64 \%$ & $50 \%$ \\
enriched by inference & $50 \%$ & $79 \%$ & $61 \%$ \\
\hline
\end{tabular}

(arround 400 categories). This experiment shows that our method is more successful in the inference of specific context types (see Table I). However, this could be applied only in our case of news reading where the content and geo-location have higher impact on the user interest and behaviour.

\section{B. Predicting readers' activity}

In this experiment we show the improvement of predicting readers' activity when context inferred by our method is used. We prepared this experiment as comparison of the prediction with and without inferred context. We made prediction using real data. We split the logs of user activity into train and test interval. Then we predicted activity from test interval only by using training interval. To calculate the success rate we compared predicted and real data from the test interval.

The prediction is made by using user activity and simulated situation. Predicting algorithm simply tries to find the best matching situation in the training set to complete its task. Training set contains pairs of condition and action. Condition includes contextual information and action is simple click on the item. We train the predictive model by clustering vectors of actions enriched by context inference. Best matching situation is then searched in discovered clusters.

We presume, when inferred context is used we get more precise prediction since more precise training data is used. To claim that prediction was correct we used news classification system used in SME. sk. This reduces the probability of wrong prediction, nevertheless, we are comparing two cases thus we are interested in improvement not in the success rate.

Table II shows that prediction with the enriched data is more successful. This experiment has shown that inferred context which has no correlation to original has positive impact on prediction of user behaviour.

\section{CONCLUSIONS}

In this paper we proposed the method for context inference which is based on the effect of correlation in user behaviour. Our method helps to semantically enrich data and fills the gap which is caused by unavailability of certain context for certain users. We showed that by context inference we enriched the process of recommendation. Secondly, we showed that we are capable of inferring contextual information correctly by using fabricated inference of original context components. Finally, we showed that using inferred context positively influences the results of user behaviour prediction. Predicting behaviour considerably improves information retrieval or recommendation since behaviour has profound effects on the information need.

Our approach combines more techniques available in data mining to infer context which is not directly available for users. We decided not to use standard association rules mining because it seriously hinders by discretized values. We work with continuous values instead of nominal or discretized. We showed how this approach is designed and what is the precision in the domain of news.

In the future work we plan to integrate our method for contextual information inference into the existing recommender system [18] [19] to research the impact on the quality of recommender system. We also plan to demonstrate our method in utilization for different domains where we are able to work with different context types and prove its generic nature. We also want to move start working with other user attributes which could be understood as context. We want to use age, gender or other demographic attributes and infer these attributes if necessary.

\section{ACKNOWLEDGEMENT}

This work was partially supported by the grants VG1/0675/11/2011-2014 and APVV-0208-10.

\section{REFERENCES}

[1] B. Mobasher, "Contextual user modeling for recommendation," in Keynote at the 2nd Workshop on Context-Aware Recommender Systems, 2010.

[2] P. M. Niedenthal, S. Kruth-Gruber, and F. Ric, "What Information Determines the Recognition of Emotion?" in The Psychology of Emotion: Interpersonal Experiential, and Cognitive Approaches. Principles of Social Psychology. Psychology Press, 2006, pp. 136-144.

[3] P. Kantor, F. Ricci, L. Rokach, and B. Shapira, Recommender systems handbook, 2010, vol. 54. [Online]. Available: http://www.lavoisier.fr/notice/fr336917.html

[4] W. Dargie, "Why is context-aware computing less successful?" Proceedings of the 5th ACM International Workshop on Context-Awareness for Self-Managing Systems, pp. 1-3, 2011. 
[5] M. Kahng and S.-g. Lee, "Ranking in context-aware recommender systems," in Proceedings of the 20th International Conference companion on World wide web. New York, New York, USA: ACM, 2011, pp. 65-66.

[6] L. Baltrunas, B. Ludwig, and F. Ricci, "Context relevance assessment for recommender systems," in Proceedings of the 15th International Conference on Intelligent user interfaces. ACM, 2011, pp. 287-290.

[7] H. Asoh, "An Analysis of Differences between Preferences in Real and Supposed Contexts," Knowledge Creation Diffusion Utilization, 2010

[8] D. Riboni and C. Bettini, "COSAR: hybrid reasoning for context-aware activity recognition," Personal and Ubiquitous Computing, vol. 15, no. 3, pp. 271-289, Aug. 2010.

[9] S. Konomi, "Colocation networks: exploring the use of social and geographical patterns in context-aware services," in Proceedings of the 13th International Conference on Ubiquitous computing. ACM, 2011, pp. 565-566.

[10] A. Cádiz, S. González, and K. Mens, "Orchestrating contextaware systems: a design perspective," in Proceedings of the first International workshop on Context-aware software technology and applications. ACM, 2009, pp. 5-8.

[11] H. Rahnama, S. Jamshidi, S. Johns, and A. Shepard, "CAMPUS: context aware mobile platform for uniformed security," in Proceedings of the 13th International Conference on Ubiquitous computing, no. Figure 1. ACM, 2011, pp. 489-490.

[12] K. Oku, S. Nakajima, J. Miyazaki, S. Uemura, H. Kato, and F. Hattori, "A Recommendation System Considering Users' Past/Current/Future Contexts," ids.csom.umn.edu, pp. 3-7, 2010.

[13] A. Komninos, A. Plessas, V. Stefanis, and J. Garofalakis, "Application of dimensionality reduction techniques for mobile social context," in Proceedings of the 13th International Conference on Ubiquitous computing. ACM, 2011, pp. 583584.

[14] M. Barla, "Towards Social-Based User Modeling and Personalization," in Proceedings of the Information Sciences and Technologies Bulletin of the ACM Slovakia. ACM, 2011, pp. 52-60.

[15] A. Said, S. Berkovsky, and E. De Luca, "Group recommendation in context," in Proceedings of the 2nd Challenge on Context-Aware Movie Recommendation. New York, New York, USA: ACM, 2011, pp. 2-4.

[16] D. Zeleník, "An Approach to Context Aware Event Reminding," in Proceedings of the Information Sciences and Technologies Bulletin of the ACM Slovakia. ACM, 2011, pp. 126-130.

[17] X. Su and T. M. Khoshgoftaar, "A Survey of Collaborative Filtering Techniques," Advances in Artificial Intelligence, vol. 2009, no. Section 3, pp. 1-20, 2009. [Online]. Available: http://www.hindawi.com/journals/aai/2009/421425.html
[18] D. Zeleník and M. Bieliková, "News Recommending Based on Text Similarity and User Behaviour," in Proceedings of the 7th International Conference on Web Information Systems and Technologies. Noordwijkerhout, The Netherland: ScitePress, 2011, pp. 302-307.

[19] M. Kompan, "Group Inspired Single-User Collaborative Filtering," in Proceedings of the Information Sciences and Technologies Bulletin of the ACM Slovakia. ACM, 2012, pp. 35-37. 